\title{
US-Studie
}

\section{Cannabis befeuert Süchte, aber keine Depressionen}

_ Mit der zunehmenden Legalisierung von Cannabisprodukten nicht zuletzt in den USA fragen sich viele, ob da nicht ein weiteres Drogenproblem die Gesundheit der Bevölkerung gefährden könnte.

Epidemiologen vom National Institute on Drug Abuse in Bethesda befragten 43.000 US-Bürger über 18 Jahren nach ihrem Drogenkonsum. Zugleich fahndeten sie mittels Fragebögen nach psychischen Störungen.

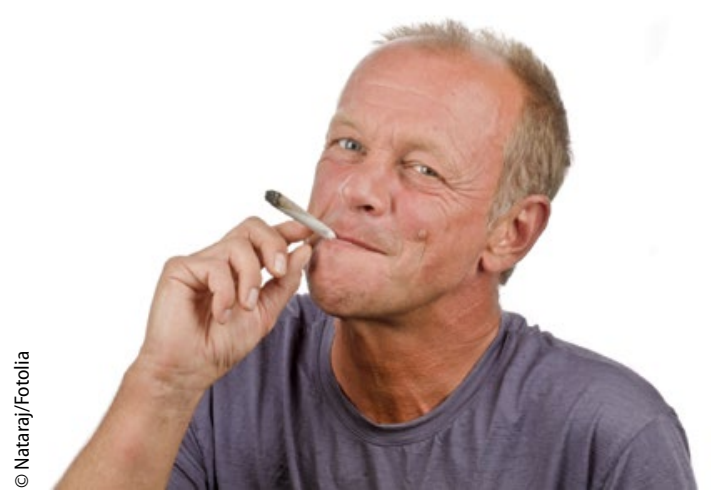

\section{Erhöhte Mortalität}

\section{Schwindel - eine Krankheit zum Tode?}

Wer an Schwindel leidet, hat ein erhöhtes Mortalitätsrisiko. Einer US-Studie zufolge sind die Schwindelsymptome ein unabhängiger Risikoparameter.

HNO-Spezialisten der Harvard Medical School in Boston, haben die Daten von über 210 Millionen US-Amerikanern durchforstet, die sich 2008 am National Health Interview Survey beteiligt hatten. Rund 11\% der Studienteilnehmer im Alter von durchschnittlich 46 Jahren hatten in diesem Jahr mindestens einmal an Schwindel gelitten. 9\% von ihnen starben in den folgenden fünf Jahren. Bei den Schwindelfreien betrug die Mor-
Insgesamt ließen sich Daten von 34.600 Teilnehmern auswerten. 1.279 von ihnen hatten in den vergangenen zwölf Monaten Cannabis konsumiert (3,7\%). Bei ihnen wurden deutlich häufiger psychische Störungen festgestellt als bei Personen ohne Cannabiskonsum. Dabei ergab sich vor allem eine erhöhte Rate von Suchterkrankungen, nicht jedoch von Depressionen, bipolaren Erkrankungen, Dysthymien oder Angstund Panikstörungen. Alkohol- und Suchterkrankungen wurden bei den Cannabiskonsumenten etwa doppelt so oft festgestellt wie bei Nicht-Kiffern.

Drei Jahre später waren Prävalenz und Inzidenz von Suchterkrankungen bei den cannabiskonsumierenden Teilnehmern weiter gestiegen. Aber weiterhin zeigte sich keine erhöhte Inzidenz für Ängste und Depressionen. - mut

- Blanco C et al. JAMA Psychiatry 2016; epub 17.2.16, doi: 10.1001/jamapsychiatry.2015.3229

\section{Migräneexperten leiden meist selbst an Migräne}

Rund 70\% aller Kopfschmerz- und Migränespezialisten (bei den Frauen sogar 86\%) leiden nach den Ergebnissen einer US-Umfrage unter Ärzten der ,American Headache Society" selbst an episodischer und $14 \%$ an chronischer Migräne. Der Anteil ist damit weit höher als in der übrigen Bevölkerung. Für diese beträgt die Lebenszeitprävalenz bei Frauen lediglich $43 \%$ und 18\% bei den Männern.

- mut

- Evans RW et al. Headache 2016; online 1. März; doi: 10.1111/head.12782

\section{Steigende Kosten durch multiresistente Erreger im Pflegeheim}

Die Kosten, die durch multiresistente Erreger in Pflegeeinrichtungen entstehen, belaufen sich im Mittel pro Heim aufjährlich rund 50.000 Euro. Nach einer Analyse der Uni Greifswald schlagen dabei vor allem Kosten für personellen Aufwand und Materialien zu Buche. - eo - BMJ Open 2016; 6:e008458

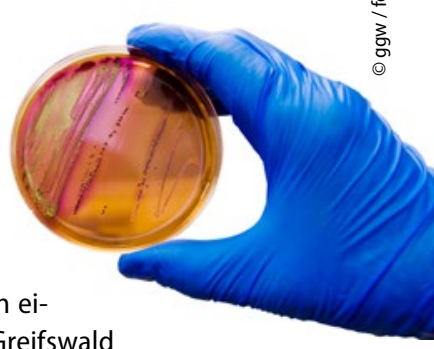

talität 2,6\%. Nach Abgleich gegen Alter und Geschlecht ergab sich aus diesen Zahlen eine schwindelassoziierte Verdoppelung der Sterblichkeitsziffern. Auch nach Berücksichtigung weiterer Faktoren wie Diabetes, kardio- und zerebrovaskulären Erkrankungen sowie malignen Tumoren war ein unabhängiger Einfluss von Schwindelsymptomen auf die Mortalität nachweisbar. Das Risiko zu sterben lag für Schwindelpatienten noch immer 70\% über dem Risiko von Schwindelfreien (OR 1,7). = rb

- Corrales CE et al. Februar; doi: 10.1002/lary.25902

\section{E-Health - Was verändert sich in der ärztlichen Praxis?}

Vernetzung, Telemedizin und das Internet, intelligente Geräten und sog. „Wearables" machen vor der Praxistür nicht halt. Von A wie Aufklärung bis Z wie Zuweisung der Patienten bildet IT schon jetzt eine Basis der medizinischen Versorgung. Sie bietet große Chancen für mehr Transparenz, bessere Kommunikation und auch für mehr Qualität.

- Terminhinweis: Frühjahrssymposium der Korporativen Mitglieder der Deutschen Gesellschaft für Innere Medizin: E-Health Was verändert sich in der ärztlichen Praxis? 10. April 2016 von 14.00 bis 16.30 Uhr Congress Center Rosengarten in Mannheim, Saal 10 